\title{
Review of: "Identification of Novel ADGRV1 and KCNC2 Variants Using Whole-Exome Sequencing in Two Colombian Patients with Usher and Encephalopathy Syndromes"
}

Jose Antonio Lopez-Escamez ${ }^{1}$

1 Universidad de Granada

Potential competing interests: The author(s) declared that no potential competing interests exist.

This is a segregation analysis in a Colombian family involving 2 rare missense variants in ADGRV1gene showing compound heterozygous inheritance associated with Usher syndrome type $2 \mathrm{C}$ in the mother and a second heterozygous de novo variant in KCNC2 gene in a child with neurodevelopment disorder with epilepsy.

The variants should be assessed according to CADD scores and ACMG guidelines and this reported in the results.

A limitation of the study is that there is no information about structural variants, including copy number variants which are very relevant in the diagnosis of neurodevelopment disorders. 